\title{
Human genetic variant E756del in the ion channel PIEZO1 not associated with protection from severe malaria in a large Ghanaian study
}

\author{
Thorsten Thye ${ }^{1 凶}$, Jennifer A. Evans ${ }^{2,3}$, Gerd Ruge ${ }^{1}$, Wibke Loag ${ }^{1}$, Daniel Ansong ${ }^{3}$, Tsiri Agbenyega ${ }^{4}$, Rolf D. Horstmann ${ }^{2}$, \\ Jürgen May ${ }^{1,5}$ and Kathrin Schuldt ${ }^{1}$
}

(c) The Author(s) 2021

Recently, a common genetic variant E756del in the human gene PIEZO1 was associated with protection from severe malaria. Here, we performed a genetic association study of this gain-of-function variant in a large case-control study including 4149 children from the Ashanti Region in Ghana, West Africa. The statistical analysis did not indicate an association with protection from severe malaria and, thus, providing evidence against a strong protective effect of the PIEZO1 E756del variant on severe malaria susceptibility.

Journal of Human Genetics (2022) 67:65-67; https://doi.org/10.1038/s10038-021-00958-2

\section{COMMUNICATION}

First evidence for a role of the human PIEZO1 variant E756 (rs59446030) in infections with Plasmodium falciparum was provided by Ma et al. [1]. Following that publication, we analyzed the common E756 variant in a large case-control study $(n=4149)$ on severe malaria (SM) in the Ashanti Region of Ghana, including 2413 SM cases and 1736 unaffected controls. This study group has proven to be a valuable resource in the past [2]. Among others, it has been used to successfully delineate the effects of established hemoglobin variants on SM subphenotypes, and it was the first study to identify a variant in the human gene ATP2B4 influencing the susceptibility to SM, which subsequently has been replicated in several other studies [2-4].

Genotyping of the PIEZO1 variant E756 (rs59446030) in the Ghanaian study group was done by allele-specific hybridization with fluorescence resonance energy transfer (FRET) in a LightCycler 480 device $[5,6]$ for all study participants $(n=4149)$ (Suppl. Table 1). The genotyping revealed the presence of the Wildtype (WT), a E756 deletion (TCC del, E756del) and, at a lower frequency, an insertion ( $\mathrm{TCC}_{\text {ins, }}$ E756ins) (Table 1). For a subset of the DNA samples $(n=10)$ the three different PIEZO1 alleles identified in our study group were validated by Sanger sequencing (Supplementary Fig. 1). Allele frequencies of E756del in the Ghanaian cases and controls were 0.19 and 0.20 , respectively, at a level similar to that of other populations of African descent [1]. Recently, Nguetse et al. described an association of the same mutation E756del in the human gene PIEZO1 with protection against severe malaria in a Gabonese case-control study [7]. The authors postulate that their results support the notion of PIEZO1 being an important host susceptibility factor for falciparum malaria. In contrast to the findings by Nguetse et al., the statistical analysis of the E756del variant did not indicate an association with protection from SM in our large Ghanaian study group.

We applied the logistic regression model as described by Nguetse et al., which tests for the effect of the heterozygous E756del genotype (WT/del) on SM compared to the homozygous E756 genotype (WT/WT), and we adjusted for sex, age, ethnicity, and $\mathrm{HbAS}$ status. The model yielded an odds ratio (OR) of 0.91 (95\% confidence interval, $\mathrm{Cl}, 0.79-1.05, p=0.23$; Table 1). Similarly, there was no evidence for an effect of the other E756del/ins genotypes on the risk of developing SM (Table 1), neither did we observe an interaction of E756 genotypes with hemoglobin types ( $\mathrm{HbS}, \mathrm{HbC})$. Weak evidence for an association with protection from SM was observed for the E756del/del genotype (OR 0,71, 95\% Cl 0.52-0.98, $p=0.04$; Table 1). However, this result should be interpreted with caution, because it is based on a relatively small difference in frequency of genotypes between cases and controls (1.1\%) and moreover the $p$ value would not be below a significance level of $(p=0.05)$ after corrections due to multiple testing.

Discrepancies between the studies may be the result of inherent geographical differences, i.e., the degree of malaria exposure, or different genetic backgrounds of ethnic groups, or sample size (Gabonese study $n_{\text {total }}=446$; Ghanaian study $n_{\text {total }}=4149$ ). In addition, in their study Nguetse et al. included mild malaria cases as controls, whereas the control group of the Ghanaian study consisted of apparently healthy children at time of recruitment [2]. As there is considerable heterogeneity of malaria-associated loci in terms of mode of effect as well as across populations and subphenotypes [8], it is important to note, that a failed replication of a marker-trait association could also indicate a populationspecific effect.

\footnotetext{
${ }^{1}$ Department of Infectious Disease Epidemiology, Bernhard Nocht Institute for Tropical Medicine, Hamburg, Germany. ${ }^{2}$ Department of Molecular Medicine, Bernhard Nocht

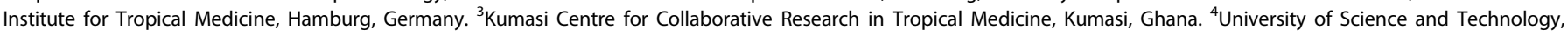

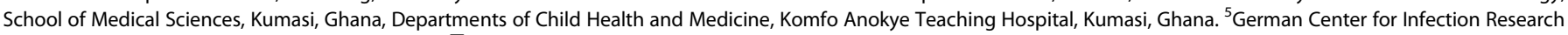
(DZIF), Hamburg-Lübeck-Borstel-Riems, Germany. ${ }^{凶}$ email: thye@bnitm.de
} 
Table 1. Association analyses of PIEZO1 E756del (rs59446030) with severe malaria in the Ghanaian case-control study

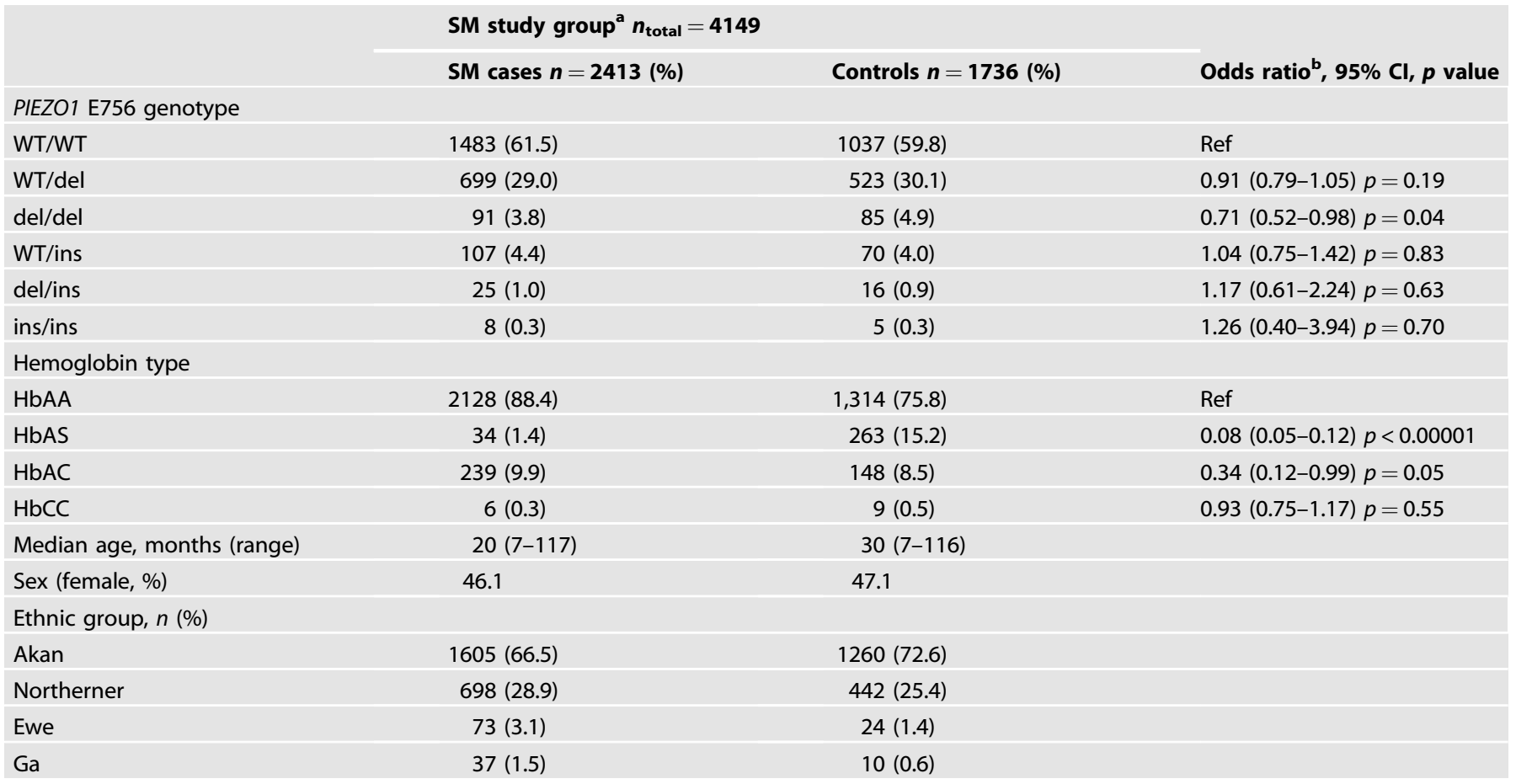

$S M$ severe malaria, $\mathrm{Cl}$ confidence interval.

aSevere malaria case group includes discrete and partly overlapping phenotypes, severe malaria anemia defined as hemoglobin level $<5 \mathrm{~g} / \mathrm{dl}$, cerebral malaria defined as Blantyre coma score $<3$, and hyperlactatemia; prostration, hyperparasitemia, respiratory distress.

${ }^{\mathrm{b}}$ Results of logistic regression adjusted for age, sex and ethnic group.

In conclusion, our results provide evidence against a strong protective genetic effect of the PIEZO1 E756del variant on SM susceptibility and underline the need for further studies in populations exposed to falciparum malaria.

\section{DATA AVAILABILITY}

Genotypes generated in this study can be retrieved from the data sharing platform ZENODO (https://zenodo.org; https://doi.org/10.5281/zenodo.4925969).

\section{REFERENCES}

1. Ma S, Cahalan S, LaMonte G, Grubaugh ND, Zeng W, Murthy SE, et al. Common PIEZO1 allele in African populations causes RBC dehydration and attenuates plasmodium infection. Cell. 2018;173:443-55.e12.

2. Timmann C, Thye T, Vens M, Evans J, May J, Ehmen C, et al. Genome-wide association study indicates two novel resistance loci for severe malaria. Nature. 2012;489:443-6.

3. May J, Evans JA, Timmann C, Ehmen C, Busch W, Thye T, et al. Hemoglobin variants and disease manifestations in severe falciparum malaria. JAMA. 2007;297:2220-6.

4. Rockett KA, Clarke GM, Fitzpatrick K, Hubbart C, Jeffreys AE, Rowlands $K$, et al. Reappraisal of known malaria resistance loci in a large multicenter study. Nat Genet. 2014;46:1197-204.

5. Lyon E, Wittwer CT. LightCycler technology in molecular diagnostics. J Mol Diagn. 2009;11:93-101. https://doi.org/10.2353/jmoldx.2009.080094.

6. Schuldt K, Ehmen C, Sievertsen J, Evans J, May J, Ansong A. et al. Lack of association of CD55 receptor genetic variants and severe malaria in Ghanaian children. G3. 2017;7:859-64.

7. Nguetse CN, Purington N, Ebel ER, Shakya B, Tetard M, Kremsner PG, et al. A common polymorphism in the mechanosensitive ion channel PIEZO1 is associated with protection from severe malaria in humans. Proc Natl Acad Sci USA. 2020;117:9074-81.
8. Malaria Genomic Epidemiology Network. Insights into malaria susceptibility using genome-wide data on 17,000 individuals from Africa, Asia and Oceania. Nat Commun. 2019;10:5732.

\section{AUTHOR CONTRIBUTIONS}

$\Pi$ and KS performed statistical analyses, $T A, R D H$, and JM designed and supervised the study, JAE, GR, WL, and DA conducted participant recruitment and data acquisition, $\mathrm{TT}$ and $\mathrm{KS}$ wrote the communication.

\section{FUNDING}

The work was funded by the German Federal Ministry for Education and Research, the German National Genome Research Network (NGFN; www.ngfn.de). The funders had no role in study design, data collection and analysis, decision to publish, or preparation of the manuscript. Open Access funding enabled and organized by Projekt DEAL.

\section{ETHICS APPROVAL}

Ethical clearance was granted by the Committee for Research, Publications and Ethics of the School of Medical Sciences, Kwame Nkrumah University of Science and Technology, Kumasi, Ghana.

\section{CONSENT TO PARTICIPATE}

All procedures were explained to parents or guardians of the participating children in the local language, and written or thumb-printed informed consent was obtained.

\section{COMPETING INTERESTS}

The authors declare no competing interests. 


\section{ADDITIONAL INFORMATION}

Supplementary information The online version contains supplementary material available at https://doi.org/10.1038/s10038-021-00958-2.

Correspondence and requests for materials should be addressed to T.T.

Reprints and permission information is available at http://www.nature.com/ reprints

Publisher's note Springer Nature remains neutral with regard to jurisdictional claims in published maps and institutional affiliations.
Open Access This article is licensed under a Creative Commons Attribution 4.0 International License, which permits use, sharing, adaptation, distribution and reproduction in any medium or format, as long as you give appropriate credit to the original author(s) and the source, provide a link to the Creative Commons license, and indicate if changes were made. The images or other third party material in this article are included in the article's Creative Commons license, unless indicated otherwise in a credit line to the material. If material is not included in the article's Creative Commons license and your intended use is not permitted by statutory regulation or exceeds the permitted use, you will need to obtain permission directly from the copyright holder. To view a copy of this license, visit http://creativecommons. org/licenses/by/4.0/.

(c) The Author(s) 2021 